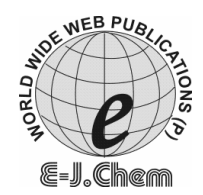

\title{
Amperometric Determination of Bismuth Using Gallacetophenone Phenylhydrazone with the Structural Elucidation of Complex
}

\author{
D.VENKATARAMANA REDDY* ${ }^{*}$ and A. VARADA REDDY \\ Department of Chemistry, S.V. University, Tirupati, India. \\ *S. G. Govt. College, PILER-517214, Chittoor (Dist.), A.P. India. \\ sggdcpiler@gmail.com
}

Received 24 December 2009; Revised 5 March 2010; Accepted 17 March 2010

\begin{abstract}
Gallacetophenone phenylhydrazone (GPPH) has been used as an analytical reagent for amperometric determination of bismuth. Bismuth is quantitatively determined by GPPH at $\mathrm{pH}$ 3.0-6.0. After studying the polarographic behaviour of GPPH and bismuth(III) at dropping mercury electrode (DME), applied potential was fixed at $-0.4 \mathrm{v} v s$. saturated calomel electrode (SCE). The method was applied for the determination of bismuth in wood's alloy. The composition of the complex corresponds to the formula $\mathrm{Bi}\left(\mathrm{C}_{14} \mathrm{H}_{14} \mathrm{O}_{3} \mathrm{~N}_{2}\right)_{2}$. The structure of the complex was arrived from the micro analytical data of the solid complex, thermogravimetric and differential thermal analysis curves and also from the infrared spectra of the complex.
\end{abstract}

Keywords: Bismuth(III)-GPPH complex, Amperometry, Elemental analysis, Thermal analysis, Structural elucidation.

\section{Introduction}

A very few analytical reagents have been employed for the determination of bismuth(III). 8-Hydroxy quinoline ${ }^{1,}$ EDTA $^{2}$ and k-morpholine-4-dithio carboxylate ${ }^{3}$ have already been reported as amperometric titrants for bismuth. Typical metal analysis has also been made on flow injection method ${ }^{4}$ in pharmaceutical products, cyclic voltammetric ${ }^{5}$, extractive spectrophotometry ${ }^{6}$, anodic stripping voltammetry ${ }^{7}$, square wave voltammetry ${ }^{8}$ and adsorptive cathodic stripping voltammetry".

Most of the selective reagents employed have hydroxyl groups e.g. pyrogallol, gallic acid and several oximes. As hydrazones and phenylhydrazones have not been employed so far, an attempt has been made to find out the usefulness of GPPH which has phenolic hydroxyl groups for the quantitative determination of bismuth(III). An attempt has also been made in elucidating the structure of bismuth-GPPH complex. 


\section{Experimental}

All chemicals used were of AnalaR Grade. Stock solution of bismuth(III) was prepared by dissolving bismuth nitrate in concentrated nitric acid and after diluting with distilled water, the solution was standardized. Solutions of other ions were prepared from their salts. An ethanolic solution of the reagent was employed and $\mathrm{pH}$ was adjusted using acetic acid and sodium acetate. A $0.2 \%$ aqueous solution of gelatin was prepared freshly before use.

Synthesis of the reagent

Gallacetophenone phenylhydrazone was prepared as per the standard procedure given by Blatt ${ }^{10}$ and Curniss et $\mathrm{l}^{11}$. A $0.01 \mathrm{M}$ reagent solution was prepared in $95 \%$ ethanol just before use.

\section{Apparatus}

A Toshniwal manual Polarograph Model No. C L -02B with inbuilt digital microammeter, A digital pH meter L I-120, A Beckmann Spectrophotometer (Model IR-18A) and Stanton Redcrafts DTA, TG and DTG simultaneous analyser model STA 780 were used.

\section{Determination of bismuth}

The polarographic behaviour of gallacetophenone phenylhydrazone and bismuth(III) at DME was studied to arrive at the suitable voltage to be fixed for the amperometric determination of bismuth. $25 \mathrm{~mL}$ of the acetate buffer ( $\mathrm{pH} 4.5$ ), $5 \mathrm{~mL}$ of $0.2 \mathrm{M}$ tartaric acid solution, $5 \mathrm{~mL}$ of $0.01 \mathrm{M}$ reagent, $1 \mathrm{~mL}$ of $0.2 \%$ gelatin and $5 \mathrm{~mL}$ of alcohol were taken in a titration cell and finally diluted to $50 \mathrm{~mL}$. Hydrogen gas was bubbled through the solution, then the drop time of the DME was adjusted to 2-3 seconds and polarogram of the reagent recorded. The polarograms of the supporting electrolyte, bismuth and the reagent are shown in Figure 1. The half wave potentials of bismuth and reagent are found to be $-0.24 \mathrm{~V} v s$. SCE and $-1.25 \mathrm{~V} v s$. $\mathrm{SCE}$ respectively. The titration was carried out by fixing the applied voltage at $-0.4 \mathrm{~V} v s$. SCE.

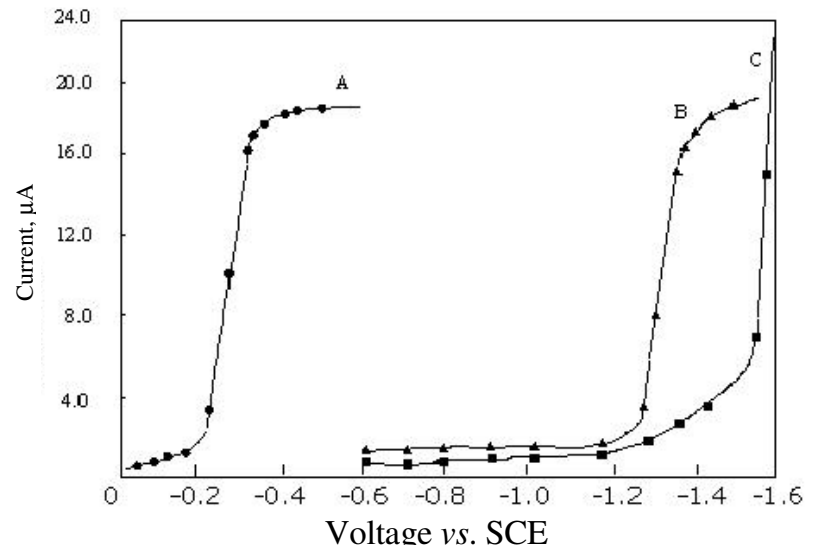

Figure 1. Polarograms of (A) Bismuth(III); (B) GPPH; (C) Supporting electrolyte ( $\mathrm{pH}=4.5$, acetate buffer)

An aliquot of standard bismuth(III) solution was transferred into the titration cell and the above contents were added keeping the volume at $50 \mathrm{~mL}$. The solution was titrated with the reagent at $-0.4 \mathrm{~V} v s$. SCE. The current readings noted were corrected for volume changes and plotted against the volume of the reagent added. An ' $L$ ' shaped curve was obtained and the end point was evaluated graphically by the extrapolation method. The procedure was repeated with known but different aliquots. The results of the some of the determinations are given in Table 1. 
Table 1. Amperometric determination of bismuth ( $\mathrm{pH} 3.0-6.0)$

\begin{tabular}{ccc}
\hline Bismuth taken, mg & Bismuth found, mg & Error, mg \\
\hline 1.99 & 2.00 & 0.01 \\
3.97 & 4.00 & 0.03 \\
5.96 & 5.96 & 0.00 \\
7.94 & 7.93 & 0.01 \\
9.93 & 9.90 & 0.03 \\
\hline
\end{tabular}

\section{Precision and accuracy}

For a set of five determinations of $3.97 \mathrm{mg}$ of bismuth, the average value found was $4.0 \mathrm{mg}$ and the standard deviation was $0.01 \mathrm{mg}$.

\section{Determination in presence of foreign ions}

To study the effect of foreign ions on amperometric determination of bismuth, 10-20 mg of various cations were added to a known volume of bismuth solution (3.97 mg bismuth) at $\mathrm{pH}$ 4.5 and determinations were carried out as mentioned above. It was observed that As(III), $\mathrm{As}(\mathrm{V}), \mathrm{Sb}(\mathrm{III}), \mathrm{Sb}(\mathrm{V})$ and $\mathrm{Pb}(\mathrm{II})$ do not interfere in the determination of bismuth in the presence of excess tartrate. $\mathrm{Cd}(\mathrm{II}), \mathrm{Se}(\mathrm{IV})$ and $\mathrm{Te}(\mathrm{VI})$ gave no precipitates with the reagent at this $\mathrm{pH}$ and hence their interference in the determination of bismuth(III) is quite unlikely. The interference of iron(III) can be avoided by adding sodium fluoride. The interference of copper(II) was avoided by precipitating $\mathrm{Cu}(\mathrm{I})$ as $\mathrm{CuSCN}$ after effecting reduction with ascorbic acid. Tin(II) was precipitated as meta stannic acid and its interference in the amperometric method is unlikely.

\section{Determination of bismuth in wood's alloy}

Bismuth has also been accurately determined in wood's alloy (Bi 50.0\%, $\mathrm{Pb} 25.0 \%, \mathrm{Sn}$ $12.5 \%, \mathrm{Cd} 12.5 \%$ ) at the above $\mathrm{pH}$ value and percentage of bismuth obtained agree with the reported values within the experimental error.

For the determination of bismuth in wood's alloy, $1 \mathrm{~g}$ of the alloy was dissolved in conc. nitric acid, evaporated, then diluted to $100 \mathrm{~mL}$ and boiled for 2 minutes. The precipitated hydrated tin(IV) oxide was filtered through whatmann-42 filter paper and washed with distilled water. The filtrate and washings were collected and made up to $250 \mathrm{~mL}$. A known aliquot of alloy solution was used to determine bismuth. Results are given in Table 2 .

Table 2. Analysis of wood's alloy

\begin{tabular}{|c|c|c|c|}
\hline \multirow{2}{*}{$\begin{array}{c}\text { Name of the } \\
\text { sample }\end{array}$} & \multirow{2}{*}{$\begin{array}{c}\text { Reported composition } \\
\%\end{array}$} & \multicolumn{2}{|c|}{ Bismuth found $\%$} \\
\hline & & Oxy iodide method. & Present method \\
\hline Wood's alloy & $\begin{array}{l}\text { Bi } 50.0 ; \mathrm{Pb} 25.0 ; \\
\text { Sn } 12.5 ; \mathrm{Cd} 12.5\end{array}$ & 49.96 & $49.88^{*}$ \\
\hline
\end{tabular}

\section{Structure elucidation of bismuth-GPPH complex}

The structure of the complex was arrived from the micro analytical data of the solid complex, thermo gravimetric and differential thermal analysis curves and also from the infrared spectra of the complex.

\section{Elemental analysis}

The elemental analysis for carbon, hydrogen and nitrogen of the bismuth - GPPH complex was recorded from Australian Micro analytical service are presented in Table 3. The results reveal that $\mathrm{Bi}(\mathrm{III})$ reacts with $\mathrm{GPPH}$ in 1.2 ratio. 
TG and DTA studies of Bi-GPPH complex

In order to arrive at the stability and structure of the metal chelate, thermogravimetric (TG) and differential thermal analysis (DTA) of the solid complex was done. The TG curves of GPPH and TG, DTA curves of the complex are shown in Figure 2 and 3 respectively.

Table 3. Elemental analysis of Bi-GPPH complex

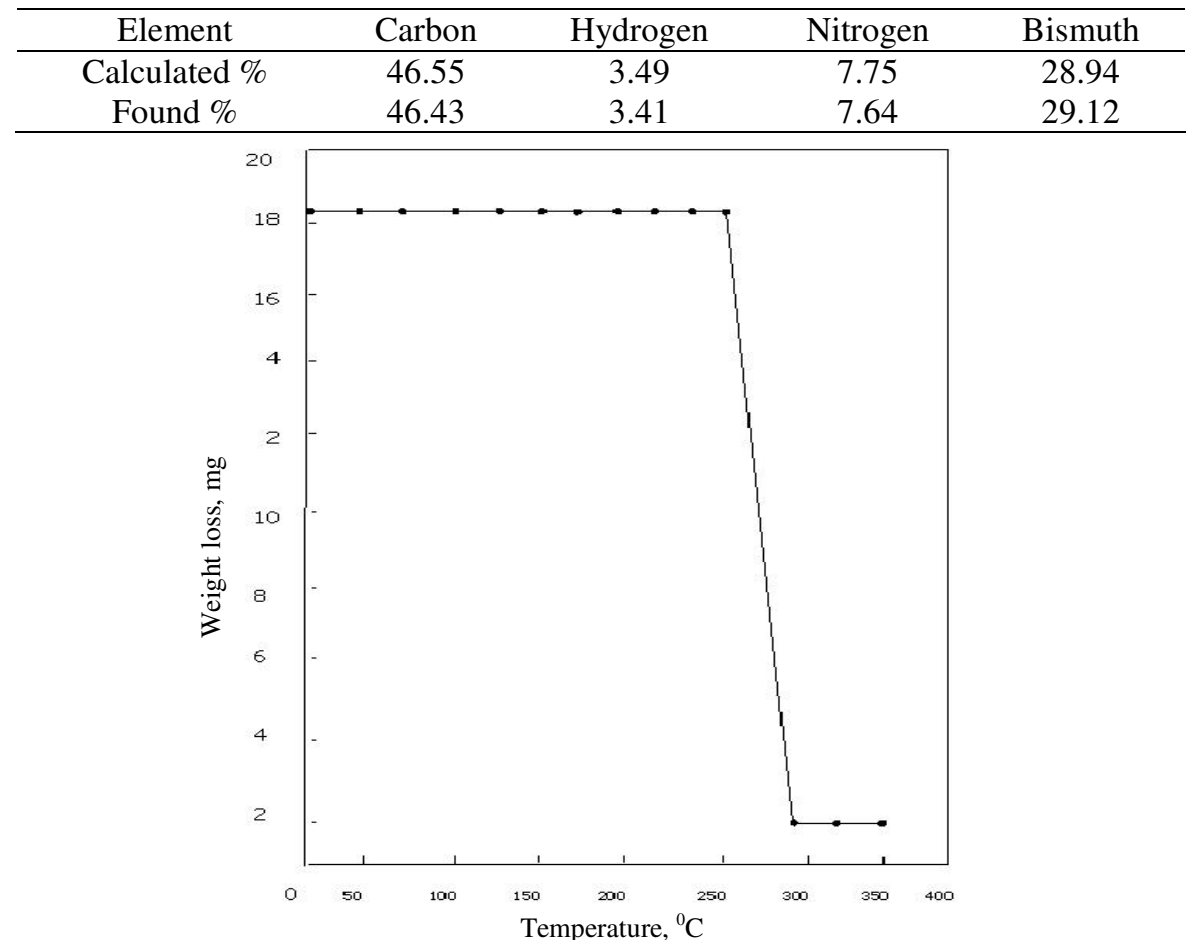

Figure 2. Thermogram of gallacetophenone phenylhydrazone

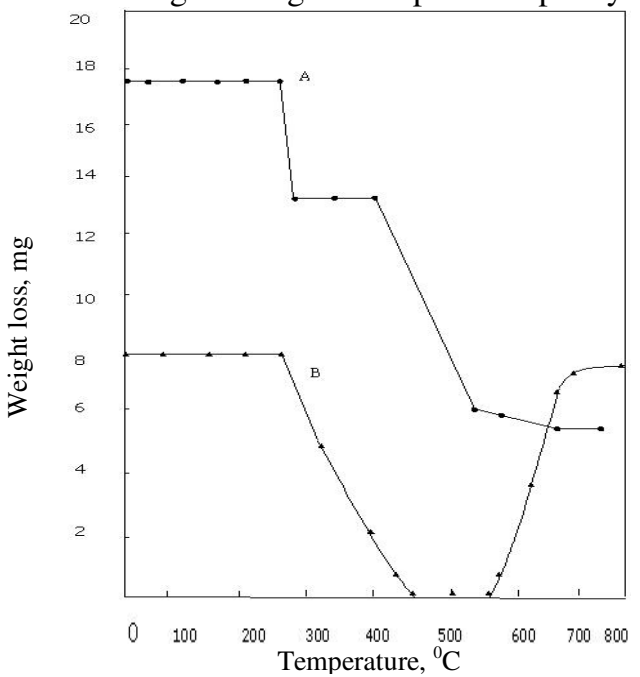

Figure 3. TG and DTA curves of Bi(III)-GPPH complex; (A) Thermogravimetric curve, (B) Differential Thermal Analysis curve 
It is seen from the above that the Bi(III)-GPPH complex starts decomposing only from $250{ }^{\circ} \mathrm{C}$ and no stepwise decomposition is observed. A stable region in the temperature range $325-375{ }^{\circ} \mathrm{C}$ is also observed. The DTA curve shows a single endothermic peak.

\section{Infrared spectral studies}

The absorption frequencies of the GPPH and bismuth-GPPH complex in infrared spectra of the solid in $\mathrm{KBr}$ are presented in the Table 4.

Table 4. Absorption frequencies $\left(\mathrm{cm}^{-1}\right)$ of GPPH and its bismuth complex and their assignments

\begin{tabular}{ccc}
\hline GPPH & Bi(III)-GPPH complex & Assignment \\
\hline $3640-3040$ & $3680-3060$ & Hydrogen bonded \\
& & O-H stretching \\
1590 & 1590 & C=N stretching \\
1320 & 1320 & O-H bending \\
(sharp) & (very small) & C-O stretching \\
1025 & 1060 & C-O \\
\hline
\end{tabular}

\section{Results and Discussion}

Bismuth shows affinity towards phenolic hydroxyl ions and it forms 1:1 complex with pyrogallol. In the present investigation, the stoichiometry of Bi(III)-GPPH complex has been found to be 1:2. If all the three hydroxyl groups are involved as in the above case, formation of 1:2 complex is quite unlikely. From the micro analytical data of the solid complex and the amperometric studies, it is found that the complex formed is $\mathrm{Bi}(\mathrm{GPPH})_{2}$.

In 8-hydroxy quinoline complexes of antimony ${ }^{12}$, scandium $^{13}$, uranium $^{14}$, and thorium ${ }^{15}$ an extra mole of oxine is also associated. The thermogravimetric curves of the above oxinates reveal that one mole of oxine is loosely held. Reddy ${ }^{16}$ has observed that an extra mole of GPPH is loosely held in uranium-GPPH complex. In the light of the above arguments, Bi-GPPH complex has been proposed this composition in analogy with uranium - GPPH complex. However, it must be stated that Bi(III) oxinate has no extra mole of oxine unlike oxinates of other metals.

The stable region corresponding to $325-375{ }^{\circ} \mathrm{C}$ of the TG curve of Bi-GPPH complex does not correspond to the loss of 1 mole of the reagent. The endothermic peak of the DTA curve indicates that the decomposition is by melting and it is continuous. Both the TG and DTA curves show that the thermal stability of the complex is fairly good.

The infrared spectra of the reagent and the complex clearly show broad peaks (3640$3040 \mathrm{~cm}^{-1}$ and $3680-3060 \mathrm{~cm}^{-1}$ respectively) corresponding to the stretching frequency of $\mathrm{OH}$ which is hydrogen bonded. This fact may be understood in terms of hydrogen bonding present between the ortho hydroxyl group and nitrogen atom of the reagent and also the complex in solid state. The absorption bands in the region $1420-1000 \mathrm{~cm}^{-1}$ which is characteristic of the $-\mathrm{OH}$ bending and $\mathrm{C}-\mathrm{O}$ stretching showed a conspicuous change in the complex when compared to the reagent.

Well defined peaks are noted in the reagent in contrast to the very small ones in the complex. The above facts therefore suggest that the complexation involves the other phenolic hydroxyls of the reagent. Further, the band due to $\mathrm{C}=\mathrm{N}$ appeared at the same frequency in the reagent as well as in the complex. This implies that the nitrogen of the $\mathrm{C}=\mathrm{N} \mathrm{g}$ roup is not involved in the complex formation. Since, the ortho hydroxyl group is 
locked in hydrogen bounding only 3, 4-hydroxyl groups of one molecule of the reagent can form bonds with bismuth. The other valency of bismuth is satisfied by its bonding probably with the 4-hydroxyl group of another molecule of the reagent. Bonding of bismuth with 2 and 3 hydroxyl groups of another molecule of the reagent may be ruled out in view of the steric factors. The high thermal stability, 1:2 stoichiometry and infrared spectral data favour the following structure for the complex.

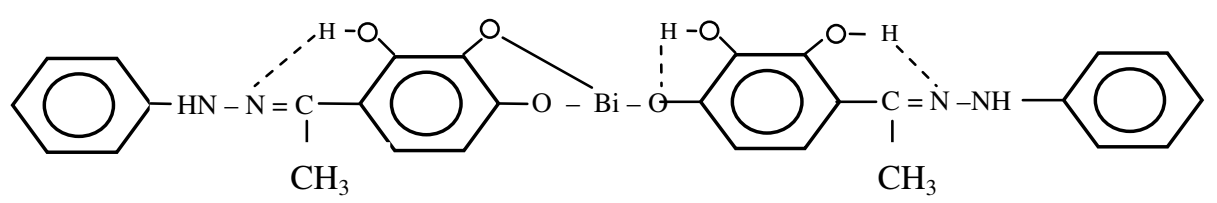

\section{Acknowledgment}

\section{Scheme 1}

The authors are grateful to Prof. C. Devendranath Reddy, S.V. University, Tirupati for his interest in this work. One of the authors (D.V. Reddy) thanks the UGC, New Delhi for awarding Junior and Senior Research fellowships.

\section{References}

1. Kolthoff I M and Lingane J J, Polarography, Vol.II Interscience, New York, 1952, 926.

2. Laitinen H A, Anal Chem., 1958, 30, 666.

3. Rao Avasrala L J, Brar Bikram S, Puri Bal K and Sethi Chaman L, Ann Chim, 1983, 73, 449.

4. Themelis D G Tzanavaras P D and Papadimitriou J, Analyst, 2001, 126(2), 247-250.

5. Chunming Wang, Qiaoyu Sun and Hulin Li, Electroanalysis, 2005, 9, 645-649.

6. Gaikwad S H, Mahamuni S V and Anuse M A, Indian J Chem Technol., 2005, 12, 365-368.

7. Gillain G, Talanta, 1982, 29, 651-654.

8. Erdo an Hasdemir and Kuddusi Karaboduk, G U Journal of Science, 2010, 23(1), 33-39.

9. Gholivand M B and Romiani, Anal Chim Acta, 2006, 571, 99-104.

10. Blatt A H, Organic Synthesis, John-Wiley Coll Vol.II 1943, 1945, 304.

11. Curniss B S, Hannaford A J, Rogers V, Smith P W G and Tatchel A R, Vogel's Text Book of Practical Organic Chemistry, $4^{\text {th }}$ Ed. ELBS and Longmans, London 1978, 886.

12. Berg R, Die Analytische Verwendung Von o-Oxychinolin (Oxin) und Sciner Derivative, $\quad 2^{\text {nd }}$ Revised Edition F Enke, Stuttgart, 1938, 3.

13. Pokras L and Bernays P M, J Am Chem Soc., 1951, 73, 7.

14. Moeller T and Ramanaiah M V, ibid 1954, 76, 5251.

15. Idem, ibid, 1953, 75, 3946.

16. Reddy K A, $O$-Hydroxyl Carbonyl Compounds as Inorganic Analytical Reagents, Ph.D., Thesis, S.V. University, Tirupati, India, 1973. 


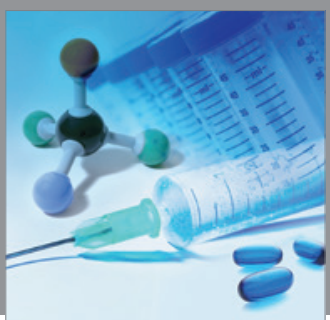

International Journal of

Medicinal Chemistry

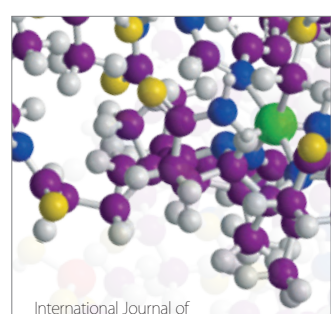

Carbohydrate Chemistry

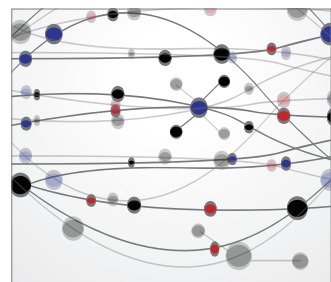

The Scientific World Journal
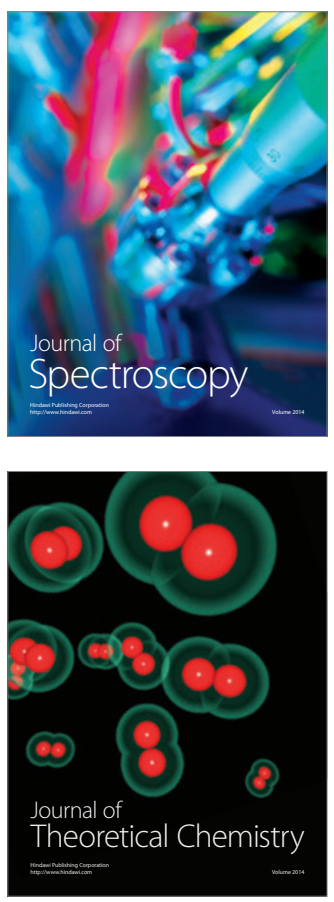
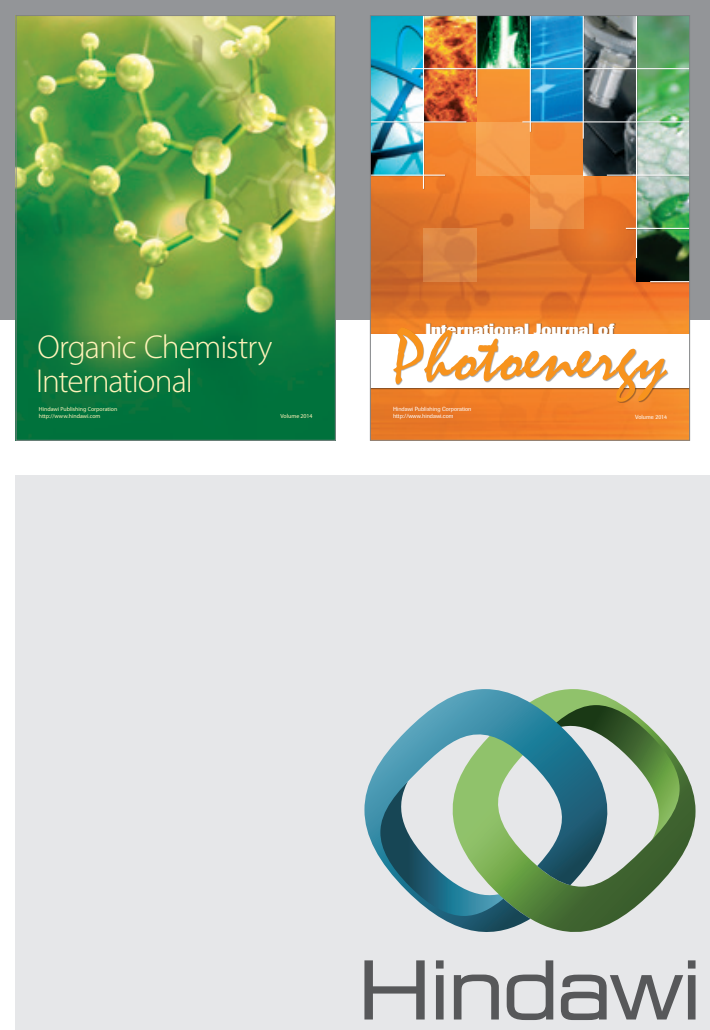

Submit your manuscripts at

http://www.hindawi.com
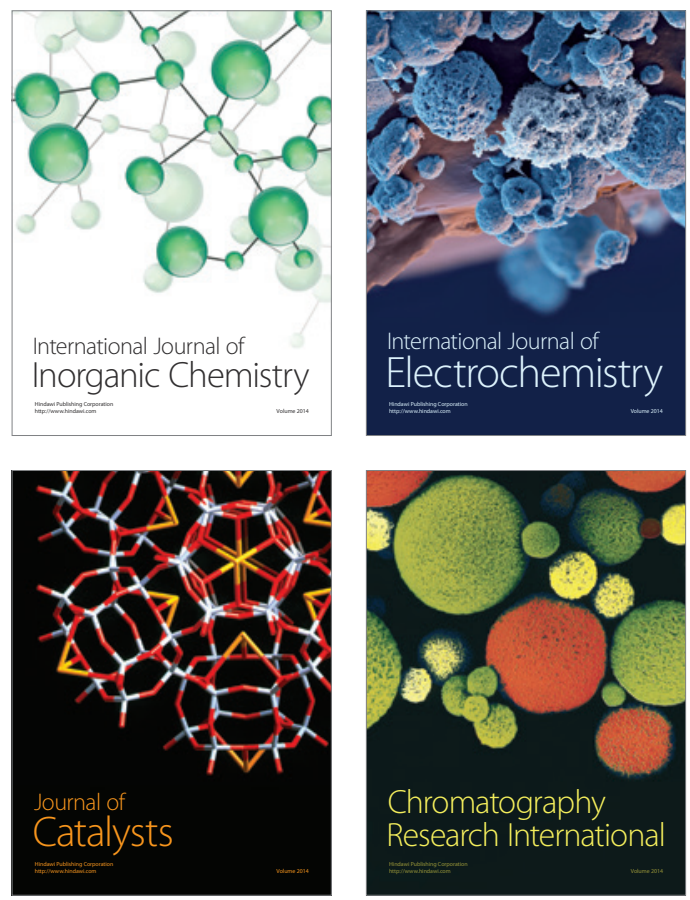
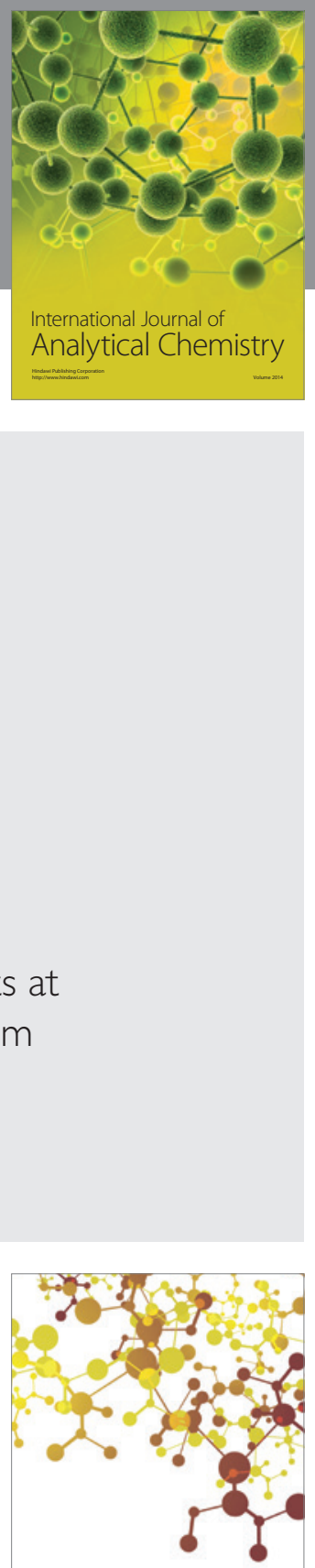

Journal of

Applied Chemistry
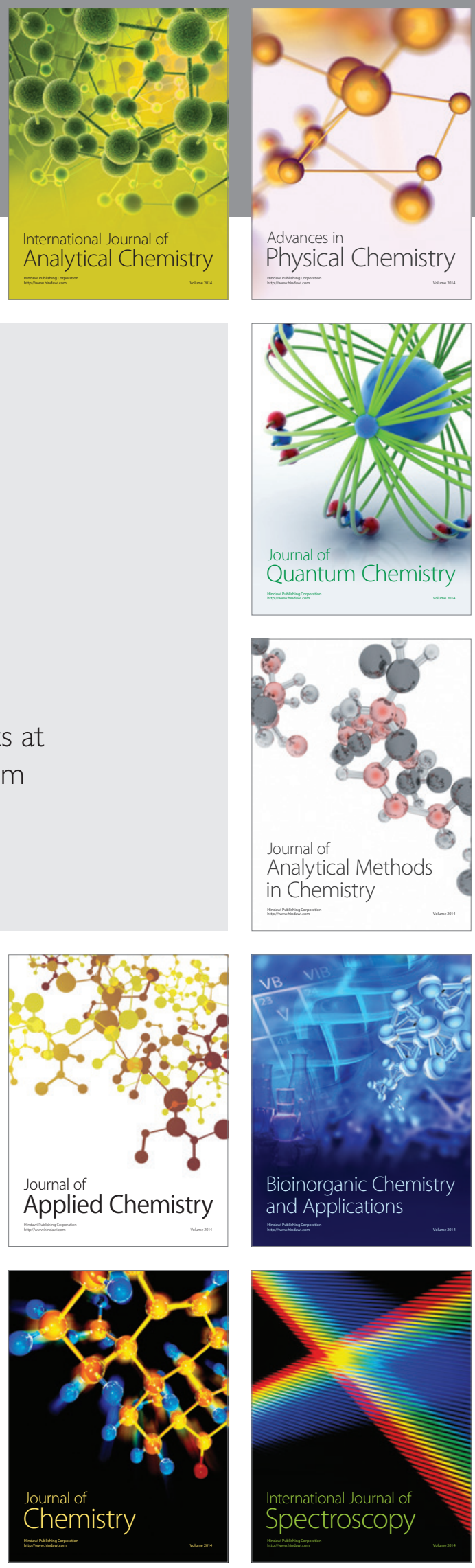